\title{
MODELAGEM ESTOCÁSTICA DA EVOLUÇÃO TEMPORAL DA MICROESTRUTURA DE SISTEMAS POLIMÉRICOS
}

\author{
T. S. M. $\operatorname{LEMOS}^{1}$, P. A. MELO Jr${ }^{1}$ e J. C. C. S. PINTO ${ }^{1}$ \\ ${ }^{1}$ Universidade Federal do Rio de Janeiro, Programa de Engenharia Química/COPPE \\ E-mail para contato: tlemos@peq.coppe.ufrj.br
}

\begin{abstract}
RESUMO - O conhecimento da microestrutura de um material polimérico é de grande interesse industrial, pois dela derivam todas as propriedades macroscópicas que definem a aplicabilidade do material. Neste trabalho, sistemas de copolimerização são modelados estocasticamente pela utilização do algoritmo de Gillespie, para determinação da evolução temporal da microestrutura dos produtos copoliméricos com arquitetura linear de cadeia. Técnicas para extensão do algoritmo de Gillespie para simulação dos processos realizados em reatores contínuos são apresentadas. São modelados os processos em reatores do tipo tanque agitado sob efeito de máxima micromistura, através do acoplamento de um módulo determinístico à simulação estocástica, para tratamento das correntes convectivas. Os processos em reatores com efeitos de macromistura qualquer, sob efeito de micromistura de segregação completa, são modelados com auxílio da discretização da distribuição de tempos de residência e convolução de simulações estocásticas em batelada.
\end{abstract}

\section{INTRODUÇÃO}

A riqueza microestrutural e conformacional dos polímeros, cada vez mais submissas a controle e manipulação, dados os avanços na síntese química, na capacidade de processamento computacional e na teoria matemática, é a principal responsável pelo sucesso dos materiais poliméricos nas mais diversas aplicações. Nesse contexto, os Processos de Polimerização Radicalar Viva/Controlada assumem grande importância, pois oferecem capacidade de produção de resinas com propriedades projetadas e bem definidas. Várias tecnologias químicas foram desenvolvidas com o intuito de estabelecer sistemas controlados, sendo as principais a Polimerização Mediada por Nitróxido (NMP), a Polimerização por Transferência de Atômica Reversível (ATRP) e a Transferência Reversível de Cadeia por Adição-Fragmentação (RAFT) (Nicolas et al., 2013). Essas tecnologias de polimerização controlada tornaram possível a produção de novos produtos poliméricos, alguns deles que se fazem interessante em toda extensão de suas cadeias, como os copolímeros do tipo multi-blocos, gradiente, graduado e de enxerto de gradiente, mostrados na Figura 1 (Pakula e Matyjaszewski, 1996; Bates e Fredrickson, 1999).

Outro fator relevante para a indústria polimérica são os reatores utilizados para a polimerização. Os processos que utilizam reatores contínuos e semibateladas são de grande importância para a indústria polimérica pelas possibilidades que podem conferir, como proporcionar a produção de determinados tipos de microestrutura e viabilizar técnica e economicamente o processo, sendo comum 


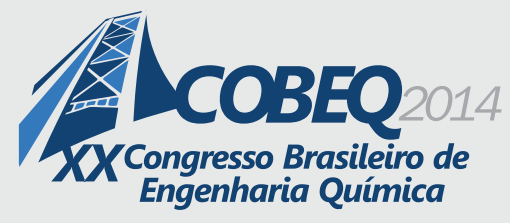

19 a 22 de outubro de 2014
Florianópolis/SC

encontrar em uso industrial vários tipos de reatores e de combinação destes, como os tanques de mistura, tubulares, tipo loop e de leito fluidizado, além dos processos em semibateladas (Asua, 2007).

Nessas circunstâncias, a modelagem matemática, a otimização e o controle dos processos de produção de produtos poliméricos assumem primordial importância, pois permitem a obtenção das distribuições das propriedades estruturais que caracterizam os materiais poliméricos, resultando na possibilidade de manipulação e projeto de produtos adequados às especificações de uso final. Porém, a obtenção das distribuições das propriedades dos polímeros, justamente por causa da riqueza estrutural e conformacional que esses sistemas apresentam, não é trivial: os métodos clássicos (resolução dos balanços de massa) padecem bastante perante o grande número de equações. Alternativas de redução de ordem (p. ex.: Método dos Momentos) mostram-se bastante úteis para caracterização das propriedades, mas admitem soluções reduzidas. Nesse contexto, os métodos estocásticos de modelagem, aqueles que fazem uso da Teoria das Probabilidades e da consideração da aleatoriedade intrínseca que os sistemas reacionais apresentam, mostram-se úteis, pois são capazes de fornecer informações sobre a microestrutura das cadeias, com possibilidade de reprodução das distribuições das propriedades microestruturais dos sistemas estudados.

\subsection{O algoritmo de Gillespie}

O algoritmo de Gillespie (Gillespie, 1976) baseia-se na simulação direta da evolução temporal do sistema através da geração de números aleatórios e fornece uma realização exata da evolução temporal do sistema. Devido à natureza do funcionamento sequencial da técnica, apresenta a possibilidade de monitorar a evolução temporal da topologia das cadeias de um sistema de polimerização. O método consiste em responder seguidamente duas perguntas: qual será o próximo evento (reação química) que ocorrerá dentro do sistema? Quando se dará esse evento?

A resposta para essas perguntas é fornecida pela função $\mathrm{P}(\tau, \mu)$, denominada função densidade de probabilidade de reação, que, multiplicada por $\mathrm{d} \tau$, fornece a probabilidade de que, dado que o sistema esteja no estado $\underline{X}(\mathrm{t})$, a próxima reação a ocorrer dentro do sistema seja a reação $\mu$, no intervalo de tempo $[t+\tau, t+\tau+d \tau)$. Essa função pode ser determinada através de uso da Teoria das Probabilidades e para um processo em batelada isotérmica, isocórica e perfeitamente misturada, sujeito a M possíveis eventos (reações químicas) é fornecida por:

$$
\mathrm{P}(\tau, \mu)=\mathrm{a}_{\mu} \exp \left[-\sum_{\mathrm{r}=1}^{\mathrm{M}} \mathrm{a}_{\mathrm{r}} \tau\right] \forall \mu=1,2, \ldots, \mathrm{M},
$$

onde $\mathrm{a}_{\mu}$ é a função de propensão da reação $\mu$ e consiste em uma medida da probabilidade da reação $\mathrm{m}$ ocorrer.

Para definir seguidamente qual reação $\mu$ ocorrerá e em que intervalo de tempo $\tau$ ela ocorrerá deve ser gerado um par de números aleatórios $\mu$ e $\tau$ em acordo com a distribuição $\mathrm{P}(\tau, \mu)$. Pelo método da inversão, pode-se mostrar que os valores de $\tau$ e $\mu$ amostrados de $\mathrm{P}(\tau, \mu)$ são fornecidos por: 


$$
\tau=\frac{1}{\sum_{\mathrm{r}=1}^{\mathrm{M}} \mathrm{a}_{\mathrm{r}}} \ln \left(\frac{1}{\mathrm{r}_{1}}\right)=\frac{1}{\mathrm{a}_{0}} \ln \left(\frac{1}{\mathrm{r}_{1}}\right) \quad ; \quad \mu=\text { menor inteiro que satisfaz }: \sum_{\mathrm{r}=1}^{\mu} \mathrm{a}_{\mathrm{r}}>\mathrm{r}_{2} \mathrm{a}_{0}
$$

sendo $r_{1}$ e $r_{2}$, números aleatórios distribuídos uniformemente no intervalo [0,1].

Assim, é possível simular etapa a etapa a evolução temporal do sistema. Após cada um dos eventos, o tempo, o estado do sistema e as funções de propensão são atualizados e novos números aleatórios são gerados para determinação do próximo evento. O procedimento é repetido até que um critério de parada para o algoritmo seja satisfeito.

\subsection{Justificativa}

Percebe-se algum avanço recente na área de síntese e caracterização de copolímeros com estrutura bem definida, quando se tem interesse em descrever a estrutura de toda a cadeia, apesar da quantidade de trabalhos na área ainda ser escassa (Al-Harthi et al., 2009; Wang e Broadbelt, 2009; Van Steenberge et al., 2012). Entretanto, não são encontradas, na literatura, investigações que utilizem reatores com modo contínuo de operação para produção desses materiais, que se fazem interessantes em toda extensão de suas cadeias poliméricas. Outro fator é a definição do algoritmo de Gillespie para, somente, processos em bateladas bem misturadas e, de maneira geral, os trabalhos que simulam processos em semibatelada não descrevem o procedimento realizado para tal. Pelos motivos acima apresentados, justifica-se este trabalho, que tem como objetivo a sintetização de modelos dinâmicos de simulação estocástica em processos conduzidos em reatores contínuos.

\section{METODOLOGIA}

Foram desenvolvidas duas estratégias para abordagem estocástica da polimerização em sistemas contínuos: uma para sistemas com macro e micromistura máximas (reator tanque de máxima micromistura) e outra para sistemas de micromistura totalmente segregados em reatores com geometria e modo de operação quaisquer.

\subsection{Tanques de máxima micromistura}

A batelada perfeitamente misturada (máxima micromistura), que o algoritmo de Gillespie trata de maneira exata, foi transformada em um reator CSTR pela inclusão das correntes convectivas do reator contínuo de modo a respeitar a máxima macromistura. Para isto, foi realizado um modelo misto, em que as correntes convectivas foram descritas de modo determinístico e acopladas à simulação das reações químicas que são descritas pelo algoritmo de Gillespie.

No modelo desenvolvido, o algoritmo de Gillespie funciona normalmente e, em intervalos de tempos $\tau$, a atualização das espécies que surgiram ou desapareceram do sistema através das correntes convectivas é feita. O cálculo da quantidade de moléculas da espécie $\mathrm{E}$ a ser transportada através de uma corrente convectiva de vazão volumétrica $\mathrm{F}$ e concentração molar $\mathrm{C}_{\mathrm{E}, \mathrm{F}}$ é fornecido por: 


$$
E=\int_{0}^{\tau} F(t) C_{E, F} N_{A} d t,
$$

Para se respeitar a macromistura do reator, a composição química das correntes de saída é tomada como idêntica à do seio do fluido no reator. É possível perceber que o reator é facilmente transformado em sistema semibatelada pelo desligamento da corrente efluente.

O modelo mostrado aqui foi aplicado ao conhecido sistema de reações em série (Equação 4) e o resultado da foi comparado à solução determinística, encontrada pela resolução numérica das equações diferenciais resultantes do balanço molar das espécies.

$$
\mathrm{A} \stackrel{\mathrm{k}_{1}}{\longrightarrow} \mathrm{B} \quad ; \quad \mathrm{B} \stackrel{\mathrm{k}_{2}}{\longrightarrow} \mathrm{C} .
$$

\subsection{Sistemas completamente segregados}

Para os sistemas contínuos com modelo de micromistura completamente segregado, a extensão do algoritmo de Gillespie foi realizada a partir da premissa de que os modelos totalmente segregados podem ser tratados como uma coleção de microreatores operando em batelada, em que cada um desses microreatores representa um elemento de fluido (Fogler, 2009).

Como os sistemas completamente segregados são constituídos por um conjunto de reatores em batelada, a conversão média do sistema pode ser encontrada pela convolução das conversões em batelada ponderadas pela distribuição de tempos de residência (DTR), como mostrado na Equação 5:

$$
\bar{X}=\int_{0}^{\infty} X(t) E(t) d t
$$

em que $\mathrm{E}(\mathrm{t})$ é a função distribuição de tempos de residência e $\mathrm{X}(\mathrm{t})$ é a conversão.

A extensão do algoritmo de Gillespie para sistemas contínuos completamente segregados foi realizada pela convolução de um número finito de simulações em batelada, como uma espécie de resolução numérica da Equação 5. São definidas frações de fluidos a serem simuladas em bateladas, sendo os diferentes tempos de bateladas obtidos por meio da DTR e os resultados das simulações somados para obtenção do produto final.

A primeira etapa é a definição de um volume amostral $\Omega$ a ser simulado. Esse volume é posteriormente divido em $\mathrm{N}$ frações amostrais a serem simuladas de maneira independente. Caso $\Omega$ seja dividido, por exemplo, em $\mathrm{N}$ amostras de tamanhos iguais, o volume $\mathrm{V}_{\text {simul }}$ de cada uma das simulações é fornecido por $\mathrm{V}_{\text {simul }}$ a a fração de material amostrada é dada por $\mathrm{f}$ :

$$
\mathrm{V}_{\text {simul }}=\frac{\Omega}{\mathrm{N}} \quad ; \quad \mathrm{f}=\frac{1}{\mathrm{~N}} .
$$


A próxima etapa do procedimento é a determinação dos $\mathrm{N}$ tempos de batelada para a simulação das amostras. Cada tempo de simulação é tomado como o tempo médio que a i-ésima fração de material permaneceu no reator:

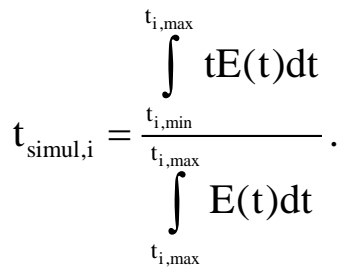

Assim, devem ser determinados esses tempos mínimos e máximos que limitam o tempo que cada fração permanece no reator para o cálculo do tempo médio, a ser adotado como tempo de simulação.

O procedimento de simulação desenvolvido foi aplicado a um tanque agitado de polimerização e também a um reator tubular. A função DTR do tanque de mistura é fornecida pela distribuição exponencial:

$$
\mathrm{E}(\mathrm{t} ; \tau)=\frac{1}{\tau} \exp \left(-\frac{\mathrm{t}}{\tau}\right)
$$

Para um reator tubular não empistonado, a DTR acumulada geralmente tem a forma de uma sigmóide. Foi utilizada a função de distribuição log-normal, por possuir a forma de sigmóide desejada, ser definida somente para o domínio positivo da variável independente e possuir forma integral (distribuição acumulada) analítica:

$$
\mathrm{E}(\mathrm{t} ; \mu, \sigma)=\left\{\begin{array}{l}
\frac{1}{\mathrm{t} \sigma \sqrt{2 \pi}} \exp \left(-\frac{1}{2}\left(\frac{\ln \mathrm{t}-\mu}{\sigma}\right)^{2}\right), \mathrm{t}>0 . \\
0, \mathrm{t} \leq 0
\end{array} .\right.
$$

\section{RESULTADOS E DISCUSSÃO}

\subsection{Tanques de máxima micromistura}

O modelo desenvolvido para reatores tanque de máxima micromistura foi implementado ao clássico sistema cinético de reações em série. O volume simulado foi de $1,0.10^{-18} \mathrm{~L}$. Foram consideradas duas correntes convectivas: uma afluente, com composição química idêntica ao estado inicial; e outra efluente, de composição idêntica à composição química no reator. Os valores dessas vazões foram considerados iguais a $20 \%$ do volume, ou seja, excessivamente alto, mas deliberadamente escolhido para tornar o teste numérico mais rápido e ilustrativo.

A evolução temporal do sistema também foi, para fins de comparação, prevista pela resolução 
das equações de taxa de reação. O resultado é mostrado na Figura 1. Nota-se que as concentrações obtidas pelo modelo apresentado oscilam em torno da concentração real.
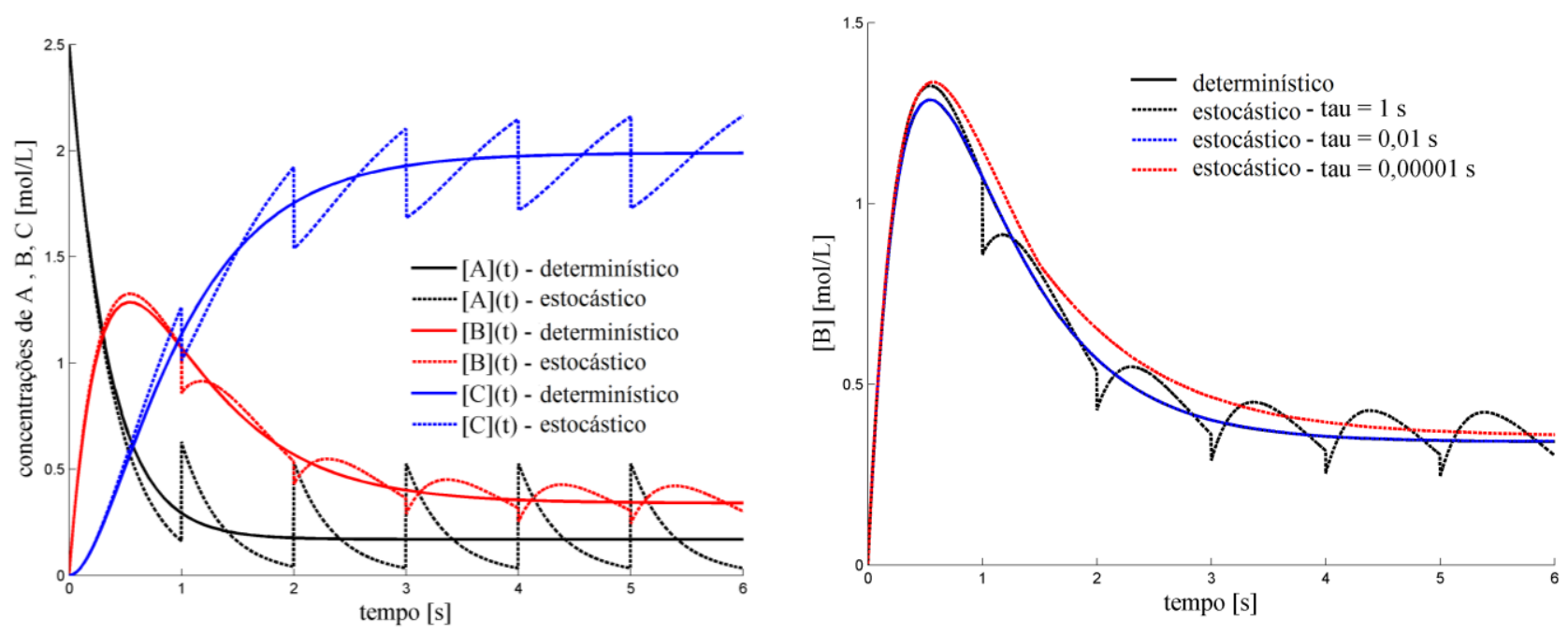

Figura 1 - À esquerda: variação temporal da concentração das espécies presentes no sistema. À direita: variação temporal da concentração da espécie $\mathrm{B}$ obtida em simulações que diferem quanto ao valor do tempo de amostragem $\tau$ ('tau': na legenda do gráfico).

É importante ressaltar que o modelo aqui apresentado constitui maneira aproximada de simulação do sistema, já que as correntes convectivas são descritas de modo peristáltico e não, como ocorre no sistema real, de forma contínua. Isto faz com que a simulação estocástica, que ocorre simultaneamente, também seja afetada, já que as probabilidades das diferentes reações são função da quantidade das espécies. Deste modo, a escolha adequada do tempo de amostragem $\tau$ seja de importância primordial (condição de salto), como pôde ser percebido na Figura 2.

\subsection{Sistemas completamente segregados}

Foram realizadas simulações para sistemas contínuos de segregação total para dois tipos de geometria de reator, tubular e tanque agitado (além da simulação em batelada) utilizando-se as funções distribuição de tempos de residência exponencial, para simulações do tanque de mistura, e log-normal, para as polimerizações em reatores tubulares. O mecanismo cinético e utilizado para as simulações foi de uma copolimerização radicalar controlada mediada por nitróxido, como pode ser encontrado de maneira pormenorizada em Lemos (2014). Ressalta-se, entretanto, que foram utilizadas cargas equimolares de monômeros e os valores utilizados para as razões de taxas de propagação foram ajustados, com intuito de formar microestruturas poliméricas do tipo gradiente (que se fazem interessantes em toda extensão das cadeias):

$$
\mathrm{r}_{1}=\frac{\mathrm{k}_{\mathrm{p} 11}}{\mathrm{k}_{\mathrm{p} 12}}=6,0 \quad ; \quad \mathrm{r}_{2}=\frac{\mathrm{k}_{\mathrm{p} 22}}{\mathrm{k}_{\mathrm{p} 21}}=0,2
$$


Os resultados das simulações são mostrados na Figura 2. É possível observar, de modo bastante claro, a influência da distribuição de tempos de residência na distribuição de tamanhos de cadeia. As distribuições de tamanhos de cadeia mostram o efeito da macromistura do reator tanque, pela exibição de uma maior dispersão nas distribuições para este sistema, como esperado, ficando o reator tubular numa posição intermediária entre o processo em batelada e o tanque de mistura. Portanto, os procedimentos propostos parecem adequados para permitir a descrição de sistemas reais de reação, em que os efeitos da DTR não podem ser desprezados.
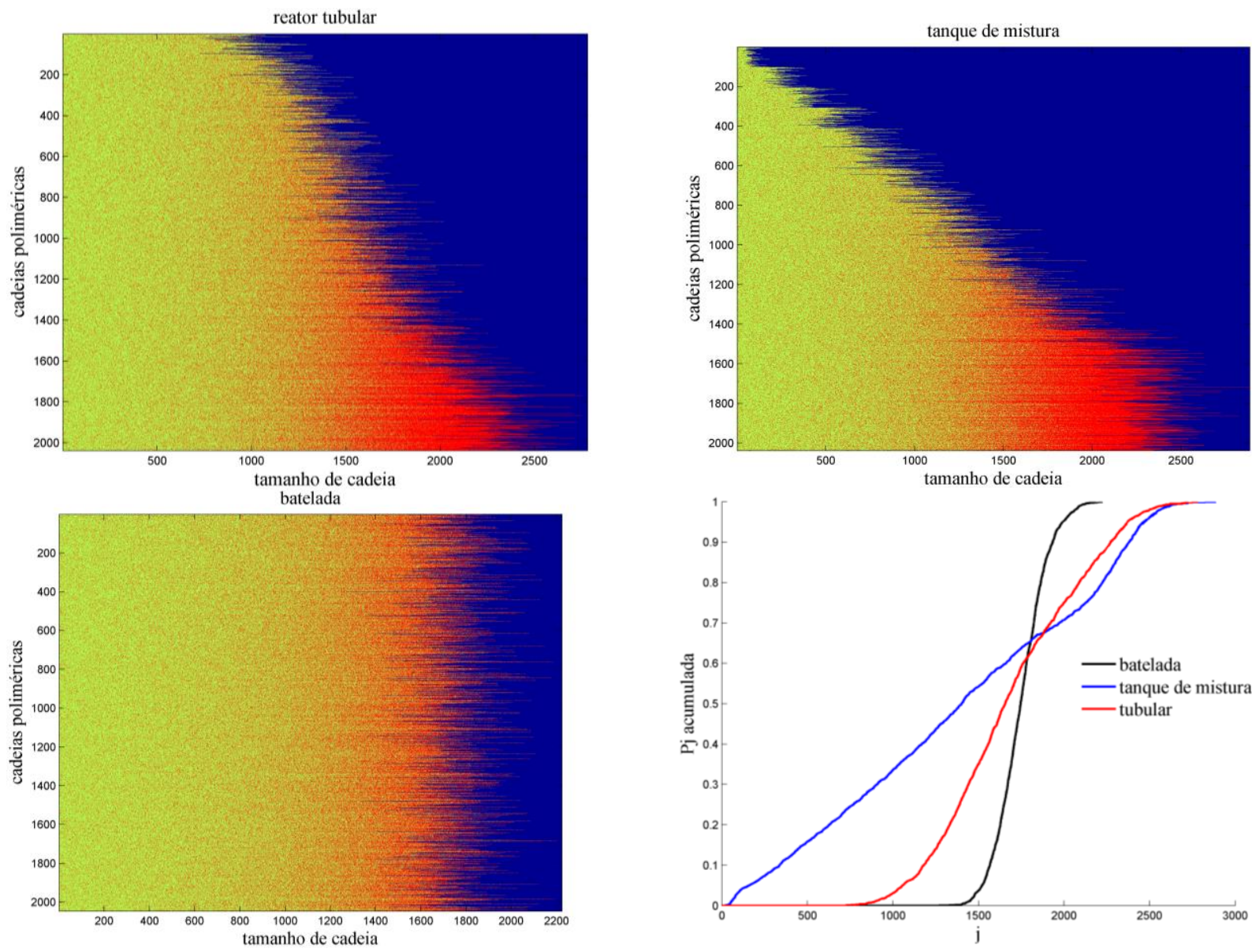

Figura 2 - Microestrutura e distribuição de tamanhos de cadeia para os produtos poliméricos formados nos diferentes reatores.

\section{CONCLUSÕES}

Foi demonstrada a vasta utilidade da modelagem estocástica para a obtenção de toda a evolução temporal da microestrutura de sistemas poliméricos. $\mathrm{O}$ desenvolvimento de uma metodologia inédita para as simulações estocásticas em reatores contínuos possui, pela importância que o processo contínuo exerce, considerável relevância para indústria polimérica. Esses modelos permitem a produção de copolímeros com sequência mérica projetada através do desenvolvimento de técnicas de otimização para a determinação das condições experimentais necessárias para a formação de 


\section{9 a 22 de outubro de 2014 \\ Florianópolis/SC}

polímeros com microestrutura projetada.

A modelagem do reator tanque de máxima micromistura constitui maneira eficiente de simulação desses sistemas. Além disso, o procedimento é válido para realização de simulações de processos em semibatelada, quê têm detalhamento de como a extensão foi feita oculto na literatura, pelo menos aplicações em sistemas de polimerização. Outro desdobramento importante foi a simulação estocástica de reatores contínuos sob efeito de micromistura completamente segregada, com modo de macromistura qualquer. $\mathrm{O}$ procedimento sugerido possui uma série de vantagens importantes. A utilização das funções de distribuição de tempo de residência é vantajosa, pois são de fácil obtenção experimental e levam em conta qualquer geometria de reator (efeito de macromistura), além de também englobar as não idealidades que se apresentam de modo frequente em problemas de engenharia de reatores, como a formação de caminhos preferenciais e volumes mortos. Por fim, a consideração de sistema de micromistura completamente segregado é, para o caso de copolimerizações, comparativamente a outros sistemas químicos, de maior aceitabilidade, já que comumente as viscosidades de sistemas poliméricos são altas, o que dificulta os processos de difusão.

\section{REFERÊNCIAS}

AL-HARTHI, M. A.; MASIHULlAH, J. K.; ABBASI, S. H.; SOARES, J. B. P. Gradient Copolymers by ATRP in Semibatch Reactors: Dynamic Monte Carlo Simulation. Macromol. React. Eng. n. 3, p. 148, 2009.

ASUA, J. M. Polymer Reaction Engineering. Oxford: Blackwell Publishing Ltd, 2007.

BATES, F. S.; FREDRICKSON, G. H. Block Copolymers - Designer Soft Materials. Phys. Today, n. 52, p. 32, 1999.

FOGLER, H. S. Elementos de Engenharia das Reações Químicas. Rio de Janeiro: LTC, 2009.

GILLESPIE, D. T. A General Method for Numerically Simulating the Stochastic Time Evolution of Coupled Chemical Reactions. Journ. of Comp. Phys., n. 22, p. 403, 1976.

LEMOS, T. S. M. Modelagem Estocástica da Formação de Produtos Copoliméricos com Microestrutura Controlada em Reatores Contínuos, M.Sc., COPPE/UFRJ, Rio de Janeiro, 2014.

NICOLAS, J.; GUILlANEUF, Y.; LEFAY, C.; BERTIN, D.; GIGMES, D.; CHARLEUX, B. Review Nitroxide-mediated polymerization, Polymer Science, n. 38, p. 63, 2013.

PAKULA, T.; MATYJASZESKI, K. Structure, thermodynamics and dynamic properties of gradient copolymers. Computer simulation. Macromol. Theo. and Simul., n. 5, p. 987, 1996.

VAN STEENBERGE, P. H. M.; D'HOOGE, D. R.; WANG, Y.; ZHONG, M. REYNIERS, M. F. KONKOLEWICZ, D.; MATYJASZEWSKI, K.; MARIN, G.B. Linear Gradient Quality of ATRP Copolymers, Macromolecules, vol.45, n. 21, pp. 8519, 2012.

WANG, L.; BROADBELT, L. Explicit Sequence of Styrene/Methyl Methacrylate Gradient Copolymers Synthesized by Forced Gradient Copolymerization with Nitroxide-Mediated Controlled Radical Polymerization. Macromolecules, n. 42, p. 7961, 2009. 